\title{
Object Oriented Assessment Of Damage Due To Natural Disaster Using Very High Resolution Images
}

\author{
Anne-Lise Chesnel and Renaud Binet \\ Commissariat à l'Energie Atomique \\ Département Analyse et Surveillance de l'Environnement \\ 91680 Bruyères-le-Châtel, France \\ Email: anne-lise.chesnel@cea.fr, renaud.binet@cea.fr
}

\author{
Lucien Wald \\ Ecole des Mines de Paris \\ Centre Energétique et Procédés \\ 06904 Sophia-Antipolis, France \\ Email: lucien.wald@ensmp.fr
}

\begin{abstract}
A building damage assessment method applied to the case of the earthquake of Bam is proposed in this paper. It uses two very high resolution images and focuses on the footprints of the buildings. The need of an accurate registration of the buildings is demonstrated; a registration method that improved the damage assessment is proposed. It allows a classification performance of the buildings among four damage grades up to $78 \%$. The impact of a lower accuracy of the buildings roofs segmentation is evaluated; we show that it mainly leads to a decrease of the capacity to identify the partial damage on buildings.
\end{abstract}

\section{INTRODUCTION}

Following a natural disaster, the local management authorities need rapidly to know the location and the extent of affected areas, along with the assessment of its impact on population and material [1].The latter need to be gradually more precise during the crisis, the post-crisis and the reconstruction. The International Charter Space and Major Disasters [2] acknowledges the usefulness of the satellite imagery to assess the impact of widespread disasters. Presently, the assessment of damage is visually conducted on images [1]. It is often difficult to detect collapsed structures with a single crisis (post-disaster) image. Accordingly, analyses rely most often on a pair of images whose dates of acquisition encompass the event of interest. The crisis image is registered and compared to the ante-disaster one which serves as a reference, in order to proceed to the comparison.

One field of interest in damage assessment is the evaluation of the damage on buildings in dense urban areas. This evaluation could be done focusing the visual analysis on the shadows of the buildings or on the debris on the ground around the buildings, but more generally it is conducted analysing the roof of the buildings. Indeed, the perception of the roofs is less affected by changes in viewing angle than other parts of a building. Moreover, they are visible by means of remote sensing and it is expected that they are less concerned with other changes. This article is focused on the analysis of damage on the roofs of the buildings. However the number of buildings being important in urban areas, such a visual analysis is time-consumming. An automated data processing could help [3].

Usually, medium resolution imagery fits the requirement of an assessment at a regional scale, but they do not allow an assessment of the performances of the methods, in absence of ground-truth. Very high resolution (VHR) images can be used for more precise results, especially concerning buildings in urban areas. Methods based on such images face new challenges [4]-[7]. The natural changes are more numerous as the resolution increases, leading to new difficulties: shadows changes, apparitions and disappearances of objects due to the human activity, seasonal changes,... In addition the relative influence of errors in registration of building roofs increases [5]. However, VHR images allow a visual quantification of the damage, hence an assessment of the quality of an automatic detection.

Our method starts from the knowledge of the buildings footprints in the reference image. Our damage assessment method, based on correlation, is applied to the case of the Bam earthquake. First, the roofs of the buildings in the reference and the crisis images are registered. Then the correlation between the pixels of the roofs is computed to assess the damage on the roofs. The buildings are classified in the last step. A reference database has been built independently and is used as a reference for registration of roofs, training for classification and assessment of the performance of the approach.

\section{IMAGES AND REFERENCE DATABASE}

The damage assessment method is applied to the case of the December 26, 2003 Bam (Iran) earthquake. The images used in this study are panchromatic VHR images from QuickBird [8] and Ikonos [9] sensors. Their dates of acquisition encompass the disaster. These sensors respectively enable spatial resolution equal to $60 \mathrm{~cm}$ and $1 \mathrm{~m}$ at nadir. These images have different acquisition parameters (table I). Three combinations of images from the two sensors acquired before and after each event are used: a pair of original QuickBird images, a pair of QuickBird images downsampled to a $1 \mathrm{~m}$ resolution, 
TABLE I

\begin{tabular}{c|ccc}
\hline Sensors & Date & $\begin{array}{c}\text { Sat. elevation } \\
\text { angle above } \\
\text { the horizon }\end{array}$ & $\begin{array}{c}\text { Sat. azimuth } \\
\text { (clockwise } \\
\text { due North) }\end{array}$ \\
\hline QuickBird & $2003-03-30$ & $78.8^{\circ}$ & $191.9^{\circ}$ \\
QuickBird & $2004-01-03$ & $63.3^{\circ}$ & $233.8^{\circ}$ \\
Ikonos & $2004-01-04$ & $62.9^{\circ}$ & $132.1^{\circ}$ \\
\hline
\end{tabular}

and a multisensor pair QuickBird/Ikonos. In the latter case, the QuickBird image is downsampled to the resolution of the Ikonos one. The image before the event is the reference image, and the image after is the crisis image.

The images are orthorectified and registered to the reference image using four ground control points (GCPs). The remaining registration error of the ground can be considered as locally constant. It is constant in the image for an area with smooth relief; steep relief can make it more varying if not accurately corrected. In addition, the images having different viewing angles, roofs of buildings are not registered precisely; as the offset of a roof is proportional to the height of the building, it can differ for each roof.

We have constructed a database to serve as a reference to assess the performances of our approach. It contains the footprints of the roofs of the buildings prior to the disaster. They were manually extracted from the reference image, and represent 2168 buildings, located in the eastern part of the town. Additionally, a damage degree is associated to each footprint of the reference database. We use the European Macroseismic Scale (EMS) damage scale as a reference scale [10]. From our own experience, we estimate that, most of the time, damage corresponding to grades 4 and 5 on the EMS can be detected in VHR images by analysing roofs, because the roofs are often affected by heavy structural damage. However a collapse of a soft storey (e.g. ground floor) is for example undetectable on the roof itself. Grade 3 can be detected at times but in a less reliable way [11], [12]. If the building is intact or if its walls are damaged but the roof seems intact, no change will be detected on the roof. Thus, four distinct EMS damage classes are retained: 0/intact, 3, 4 and 5. An extract of the Bam QuickBird reference image on which the footprints of the buildings have been overlaid is presented in figure 1 .

\section{DAMAGE ASSESSMENT WITHOUT SPECIFIC REGISTRATION OF THE BUILDINGS ROOFS}

In this part, we apply a damage assessment approach based on correlation, the images being registered as explained before using a set of four GCPs. We compute the correlation coefficient between the pixels held in the roofs in the reference and in the crisis images. In the reference image, the footprint is specified by the manual segmentation; in the crisis image, the pixels that are considered are the correspondence in terms of geographical coordinates. The buildings are then classified among the four detectable damage classes. The Support Vector Machine (SVM) is used for the classification [13], [14]. We have chosen a supervised classification for the following reasons: we consider that we have no a priori knowledge

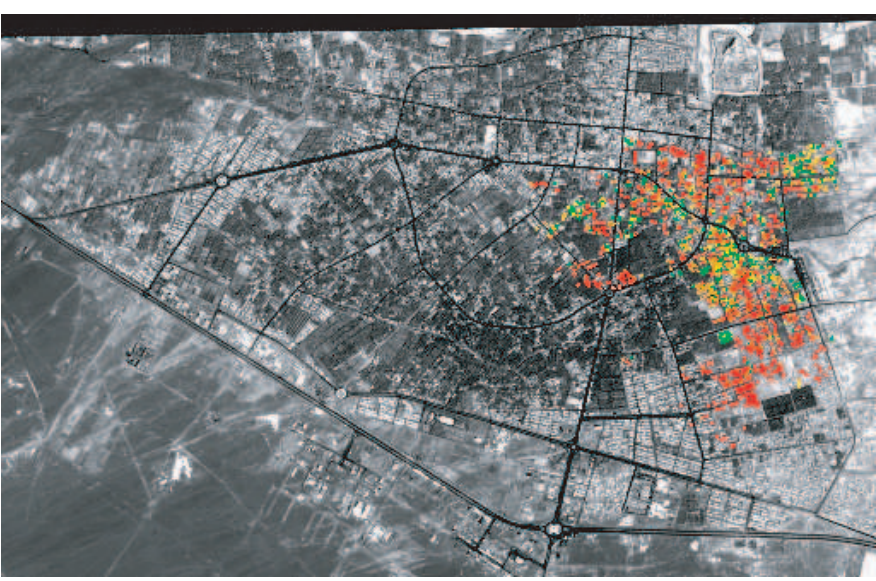

(a)

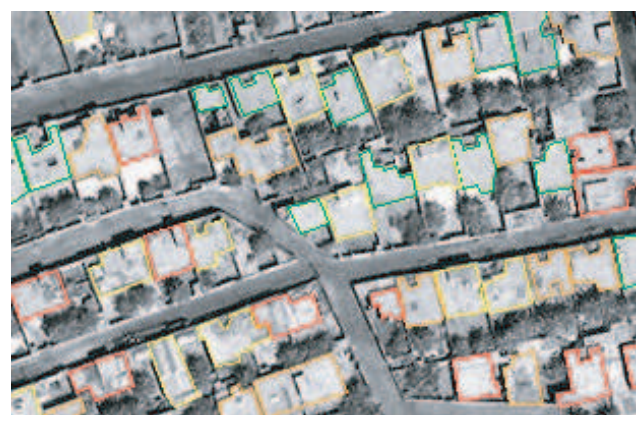

(b)

Fig. 1. QuickBird reference image (before the earthquake) of Bam. The buildings footprint of the corresponding reference database is overlaid. (a): entire scene; (b): image extract. Colour legend of the database: Green $=$ damage grade 0 ; Yellow $=$ damage grade 3 ; Orange $=$ damage grade 4 ; Red $=$ damage grade 5. Image: copyright DigitalGlobe 2003.

about the number and the different damage degrees that are present for a given disaster; moreover, for a given damage degree, the damage feature (here the correlation coefficient) can differ from one test-case to another, according to the type of buildings, to the atmospheric conditions, to the acquisition parameters, etc. To meet the requirement of an operational scope, the required training set is constrained to be small, with five examples per damage grade. In our case, these examples are randomly selected among the reference database. Finally, because the classification result depends more or less on the chosen training patterns, 100 classification tests are conducted for each case with different training sets, to ensure the robustness of the results.

For the test with the pair of original QuickBird images, the mean classification performance without roof registration is $63 \%$, the best performance being $70 \%$. The intact and the damage grade 5 represent by far the best classified buildings. The corresponding confusion matrix shows that this classification do not allow to detect the damage grade 3; this grade is totally ignored. The registration being not accurate, it induces background changes that prevents us to detect the small damage on the roofs that correspond to intermediate damage grade. 
For the test with the downsampled QuickBird images, the mean classification performance is equal to $60 \%$. Finally, for the test with the QuickBird/Ikonos pair, 53\% of the buildings are well classified. As before, the damage grade 3 is not detected. Moreover, the EMS 4 damage grade is not accurately detected, particularly for the QuickBird/Ikonos pair.

\section{DAMAGE ASSESSMENT With REgistRation OF THE BUILDINGS ROOFS}

In addition to the registration of the images, we propose to register each building roof precisely.

\section{A. Roof registration}

Our registration method estimates the maximum a posteriori of the correlation computed on the group of pixels held in the roof footprints in both images. This time, the footprint defined in the reference image is translated in the crisis image by a quantity $\vec{o}$ estimated by the algorithm of roofs registration (equation 1). Let $R_{1}$ and $R_{2}$ be respectively the reference and the crisis images with zero mean. Let $c$ and $l$ be respectively the column and the line coordinates, and $k_{c}$ and $k_{l}$ be respectively the shift value of the footprint of the roof in the crisis image along the columns and along the lines.

$$
\begin{aligned}
\vec{o}= & \arg \max _{k_{c}, k_{l}}\left\{\frac{\sum_{c, l} R_{1}(c, l) \cdot R_{2}\left(c+k_{c}, l+k_{l}\right)}{\sqrt{\sum_{c, l} R_{1}^{2}(c, l)} \cdot \sqrt{\sum_{c, l} R_{2}^{2}\left(c+k_{c}, l+k_{l}\right)}}\right\} \\
& \text { for }\{c, l \mid H(c, l)=1\}
\end{aligned}
$$

$$
\text { where } H(c, l)= \begin{cases}1 & \text { if } I_{1}(c, l) \in \text { roof footprint } \\ 0 & \text { otherwise }\end{cases}
$$

The function $H(c, l)$ may be defined by the means of a segmentation or an extraction of buildings footprint. In our case, its values are assigned by the means of the reference database of buildings. The position $\left(k_{c}^{*}, k_{l}^{*}\right)$ of the maximum correlation yields the offset $\vec{o}$.

The offset $\vec{O}$ can be written as the sum of a ground offset $\overrightarrow{o_{g}}$ due to the ground misregistration and a height offset $\overrightarrow{o_{h}}$ due to the unknown height of the building: $\vec{O}=\overrightarrow{o_{g}}+\overrightarrow{O_{h}}$. Let $\overrightarrow{o_{\perp}}$ be such that $\overrightarrow{o_{g}} \wedge \overrightarrow{o_{\perp}}=\overrightarrow{0}$. The ground offset $\overrightarrow{o_{g}}$ has no expectable orientation. On the contrary, the height offset $\overrightarrow{o_{h}}$ is solely dependent on the viewing angles for both images and on the height of the building. For intact buildings, this offset is observed in the epipolar direction $\theta$ given by the equation (2).

$$
\theta=\arctan \left\{\frac{\tan e_{1} \sin a_{2}-\tan e_{2} \sin a_{2}}{\tan e_{1} \cos a_{2}-\tan e_{2} \cos a_{2}}\right\}
$$

where $a_{1}, e_{1}, a_{2}, e_{2}$ are respectively the satellite azimuth and elevation angle above horizon of the reference and crisis images (table I).

Equation (1) is applied to a limited search area. The height offset $\overrightarrow{O_{h}}$ is due to the height of the constructions, so its amplitude differs for each building. On the contrary, we will suppose that the ground offset does not vary much. These differences are exploited to define an unique search area. Note that a 2D search is necessary due to the unpredictability of the ground offset, reducing the relevance of an epipolar image projection. The search interval is set along the $\overrightarrow{O_{\perp}}$ axis as a function of the expected registration error of the ground between the images, and along the $\overrightarrow{o_{h}}$ axis as a function of the estimated maximum height of all the buildings.

Using this registration method, the mean roof offset for all the buildings of the reference database is estimated to be between 0.2 and to 0.4 pixels, the images being registered according to the ground. As expected through a visual assessment, this is a small subpixel offset.

\section{B. Damage assessment}

The roofs being registered, the damage is assessed for each building using the correlation coefficient corresponding to the best offset. The classification uses the same SVM scheme as before.

As a mean result, $72 \%$ of the extracted buildings are well classified among the four damage grades using the pair of QuickBird images; the maximum performance is $78 \%$. Whereas the classification performance of the buildings corresponding to damage grade 0 and 5 remains stable comparing to the damage assessment without roof registration, the classification of the buildings with intermediate damage grade (3 and 4) are greatly improved, inducing a significative improvment of the global classification results. The same observation is done using the 1-m resolution images: $69 \%$ of the buildings are correctly classified using the QuickBird 1-meter downsampled images, and 62\% using the multi-sensor QuickBird/Ikonos pair of images. Moreover, for this resolution, we also observe a clear improvement of the classification of the intact buildings.

We show through this example that the quality of the registration of the roofs controls the quality of the damage assessment, even if the shifts between the roofs in the two images are small (lower than 0.5 pixel in our case). This is particularly true for the slight/medium damage.

\section{DAMAGE ASSESSMENT WITH DEGRADED ROOF SEGMENTATION}

In this section, the impact of the quality of the image segmentation on the damage assessment is investigated. Each building footprint of the reference database is defined by a polygon whose vertices are given by coordinates in the reference image. We simulate a degraded roof segmentation by randomly moving each vertices of each polygon by a different quantity that follows a normal distribution with zero mean. Three different values of variance for the distribution of this deviation are tried: 1, 3 and 5 pixel(s) (figure 2). The buildings are classified according to the correlation coefficient computed between the pixels held in the degraded footprint in the reference image and the pixels held in the degraded footprint shifted in the crisis image by the best offset estimated by the proposed registration methodology. 


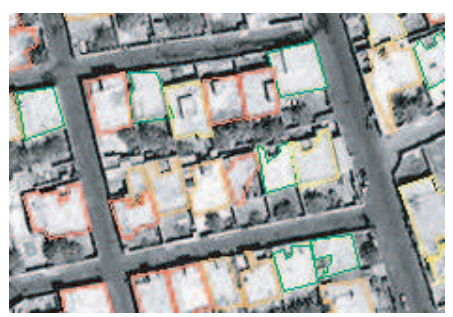

(a)

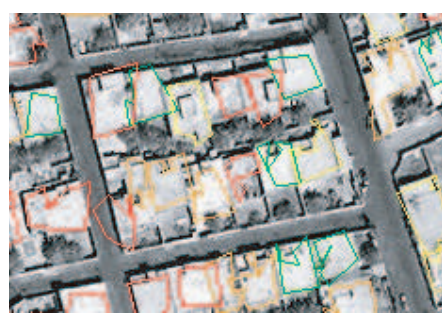

(b)

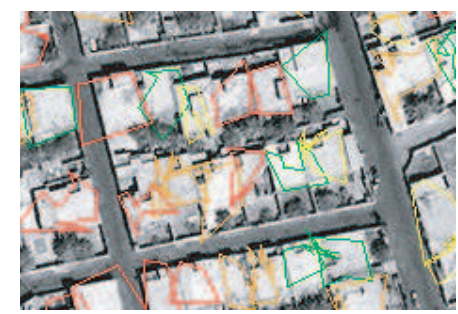

(c)

Fig. 2. The degraded buildings footprint overlaid on the QuickBird reference image of Bam. The vertices of the footprints are moved by a quantity that follows a normal distribution with a variance of (a): 1 pixel; (b): 3 pixels; (c): 5 pixels. Image: copyright DigitalGlobe 2003.

With a random deviation that follows a distribution with a variance of 1 pixel, the mean global classification is $71 \%$ and the best performance is $76 \%$. This result is roughly the same as with the original data. The conclusion is that a small error in the shape of the building extraction (figure 2(a)) do not affect the damage assessment. Considering a deviation with variance equal to 3 pixels, the mean damage assessment performance is $69 \%$, the best performance being $75 \%$. When the variance is 5 pixels, this mean performance drops to $66 \%$, with a maximum performance equal to $73 \%$.

We conclude that a lower accuracy of the shape of the extracted segments only leads to slightly lower performance of the damage assessment. Actually, a precise analysis of the results shows that the intermediate damage grades are responsible of the decrease of performance, notably the EMS damage grade 3. Indeed, the degradation of the footprint accuracy makes that, on one hand pixels outside of the buildings roof (typically at the building base) that are prone to not pertinent changes are considered in the correlation computation; and on the other hand, a part of the pixels inside the roof are ignored. Thus, the $3 \mathrm{rd}$ grade corresponding to slight damage whose magnitude is comparable to other changes not related to damage such as shadows or objects around the buildings, it is less efficiently detected.

\section{CONCLUSION}

We show in this paper the importance of the registration for a change analysis, and how it can be critical for buildings damage detection when using very high resolution images. This is illustrated with QuickBird images of the town of Bam (Iran); we aim to assess the damage on the buildings following the earthquake of December 26, 2003.

A first damage assessment is conducted after registering the images with a set of GCPs, a DTM and the RPC associated to the data. Then, a second damage assessment is proposed with a preliminary precise registration of the buildings. Although we have no information about the height and the form of the buildings, we propose a registration method that allows a subpixel registration of the buildings roofs: it leads to a clear improvement of the damage assessment.

We finally show that the quality of the extraction of the buildings impacts the damage assessment that follows. The lower the accuracy of the building extraction, the lower the damage assessment performances. However, we have shown that moderate segmentation error do not affect dramatically the performance. The mainly impacted damage grade is the $3 \mathrm{rd}$ EMS damage grade, because it corresponds to damage with small extent that can be excluded of the degraded buildings footprint and thus of the damage assessment.

We conclude that, in our opinion, the critical aspect of change analysis with very high resolution images is the precise registration of the data. This step should not be ignored, because it greatly impacts the quality of the final analysis.

\section{REFERENCES}

[1] B. Allenbach, R. Andreoli, S. Battiston, C. Bestault, S. Clandillon, and K. Fellah, "Rapid eo disaster mapping service: Added value, feedback and perspectives after 4 years of charter actions," in IEEE Proceedings of International Geoscience And Remote Sensing Symposium 2005, vol. 6, July 2005 , pp. 4373-4378.

[2] International-Charter, "International charter Space and Major Disasters," 2007, available online at: http://www.disasterscharter.org/ (accessed 15 February 2007)

[3] H. Yésou, Personnal communication, 2005.

[4] B. Adams, C. Huyck, B. Mansouri, R. Eguchi, and M. Shinozuka, "Application of high-resolution optical satellite imagery for post-earthquake damage assessment: The 2003 boumerdes (algeria) and bam (iran) earthquakes," Research Progress and Accomplishments 2003-2004, Buffalo: MCEER, 2004, http://mceer.buffalo.edu/publications/resaccom/04SP01/12_Eguchi.pdf.

[5] G. Bitelli, R. Camassi, L. Gusella, and A. Mongnol, "Image change detection on urban area: the earthquake case," in XXth ISPRS Congress, Istanbul, Turkey, July 2004, p. 692.

[6] Z. Chen and T. C. Hutchinson, "Urban damage estimation using statistical processing of satellite images: 2003 bam, iran earthquake," Proceedings SPIE, vol. 5667, pp. 289-300, 2005.

[7] M. Sakamoto, Y. Takasago, K. Uto, S. Kakumoto, Y. Kosugi, and T. Doihara, "Automatic detection of damaged area of iran earthquake by high-resolution satellite imagery," in IEEE Proceedings of International Geoscience and Remote Sensing Symposium 2004, vol. 2, September 2004, pp. 1418-1421.

[8] DigitalGlobe. Quickbird specifications. [Online]. Available: http://www.digitalglobe.com/about/quickbird.html

[9] SpaceImaging. Ikonos specifications. [Online]. Available: http://www.spaceimaging.com/products/ikonos/index.htm

[10] G. Grünthal, R. Musson, J. Schwarz, and M. Stucchi, L'Echelle Macrosismique Europenne 1998. Cahiers du Centre Europen de Godynamique et de Sismologie, Luxembourg, 2001, vol. 19.

[11] F. Yamazaki, K. Kouchi, M. Matsuoka, M. Kohiyama, and N. Muraoka, "Damage detection from high-resolution satellite images for the 2003 boumerdes, algeria earthquake," in 13th World Conference on Earthquake Engineering, International Association for Earthquake Engineering, Vancouver, British Columbia, Canada, 2004, p. 13.

[12] F. Yamazaki, Y. Yano, and M. Matsuoka, "Visual damage interpretation of buildings in bam city using quickbird images following the 2003 bam, iran, earthquake," Earthquake Spectra, vol. 21, no. S1, pp. S329-S336, December 2005 .

[13] V. N. Vapnik, The nature of statistical learning theory. New York, NY, USA: Springer-Verlag New York, Inc., 1995.

[14] C. J. C. Burges, "A tutorial on support vector machines for pattern recognition," Data Mining and Knowledge Discovery, vol. 2, no. 2, pp. 121-167, 1998. 